\section{Eosinophilic granulomatosis with polyangiitis (Churg-Strauss) induced by immune checkpoint inhibitors}

We read with great interest the articles by Kostine et $a l^{1}$ (and subsequent correspondence by Arnaud et $a l^{2}$ ) and by Belkhir et al. ${ }^{3}$ The prognosis of various cancer types has dramatically improved since the advent of immune checkpoint inhibitors (ICIs). Yet, ICI therapy is associated with frequent and potentially organ or life-threatening immune-related adverse events (irAEs), generally mimicking autoimmune or inflammatory conditions. ${ }^{4}$ Rheumatic disorders have been reported in this setting, mainly rheumatoid arthritis, polymyalgia rheumatica and systemic lupus erythematosus. ${ }^{1-3}$ Vasculitis seems to occur more seldom, with predominantly medium-vessel to large-vessel involvement. ${ }^{5}$ Here, we report on a patient with eosinophilic granulomatosis with polyangiitis (EGPA, formerly Churg-Strauss syndrome) following treatment with ICI for a stage IV melanoma.

A 34-year-old non-smoking female patient with stage IV melanoma was treated with ipilimumab (a monoclonal antibody targeting the cytotoxic T-lymphocyte associated antigen 4) and nivolumab (a monoclonal antibody targeting the programmed death protein 1) every 3 weeks. After three infusions of combined therapy, the patient developed a severe cough associated with progressive shortness of breath due to bronchial hyper-reactivity. Biological tests showed marked hypereosinophilia (up to $2.2 \mathrm{~g} / \mathrm{L}$ ) and high IgE level (763 IU/L). An extensive diagnostic work-up (including HIV, toxocarosis and aspergillosis serologies, tryptase and vitamin $\mathrm{B}_{12}$ dosages) was unremarkable. Antineutrophil cytoplasmic antibody (ANCA) serology was negative. T-lymphocyte phenotyping showed no aberrant (eg, $\mathrm{CD}^{-} \mathrm{CD}^{+}$) phenotype and no clonal T-cell receptor gene pattern was evidenced. Troponin and brain natriuretic peptide (BNP) levels as well as ECG, echocardiography and cardiac MRI were normal. Her condition improved rapidly after treatment with inhaled corticosteroids (ICSs) and long-acting beta-agonist therapy (LABA). Routine PET imaging showed a dramatic tumour response.

After four additional infusions of nivolumab maintenance therapy, the patient had recurrence of dry cough, shortness of breath, wheezing, decreased oxygen saturation (93\%) and hypereosinophilia up to $1.76 \mathrm{~g} / \mathrm{L}$. A high-resolution chest CT showed ground-glass opacities of the right lung apex and bronchial wall thickening. Bronchoscopy and bronchial biopsies were unremarkable, yet bronchoalveolar lavage could not be performed due to the bronchospasm. Nivolumab was paused and the patient's condition improved within days. When nivolumab was reintroduced, eosinophil level increased again, and asthma symptoms worsened despite of continuation of ICS/LABA, resulting in definitive discontinuation of nivolumab (figure 1). Two months after stopping nivolumab, the patient developed joint pain and swelling of both knees, whose aspiration revealed 8100 leucocytes $/ \mathrm{mm}^{3}$ (24\% of which were granulocytes) without evidence of bacterial infection nor microcrystalline arthropathy and negative immunological work-up (including ANCA, rheumatoid factor, antinuclear and anticitrullinated protein autoantibodies). Concomitantly, brain MRI performed for melanoma follow-up showed evidence of pansinusitis (mucosal thickening and opacification of sinuses). Hence, in the context of asthma, hypereosinophilia, pulmonary infiltrates, arthritis and paranasal sinus abnormalities, the diagnosis of EGPA (formerly ChurgStrauss syndrome) was retained ${ }^{6}$ and the patient was treated with ICS/LABA and intraarticular corticosteroid injection.

Moderate asymptomatic hypereosinophilia has been reported in up to $3 \%$ of patients treated with ICI. $^{7}$ To our knowledge, this is the first case report of a patient with ICI-induced EGPA. Since the patient showed no evidence of genuine vasculitis, one might also classify him as having hypereosinophilic asthma with systemic manifestations. ${ }^{8}$ The pathophysiological process leading to eosinophilia remains unknown, but the high level of serum IgE strongly suggests Th2-mediated reactive hypereosinophilia induced by ICI.

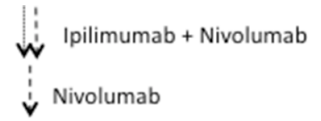

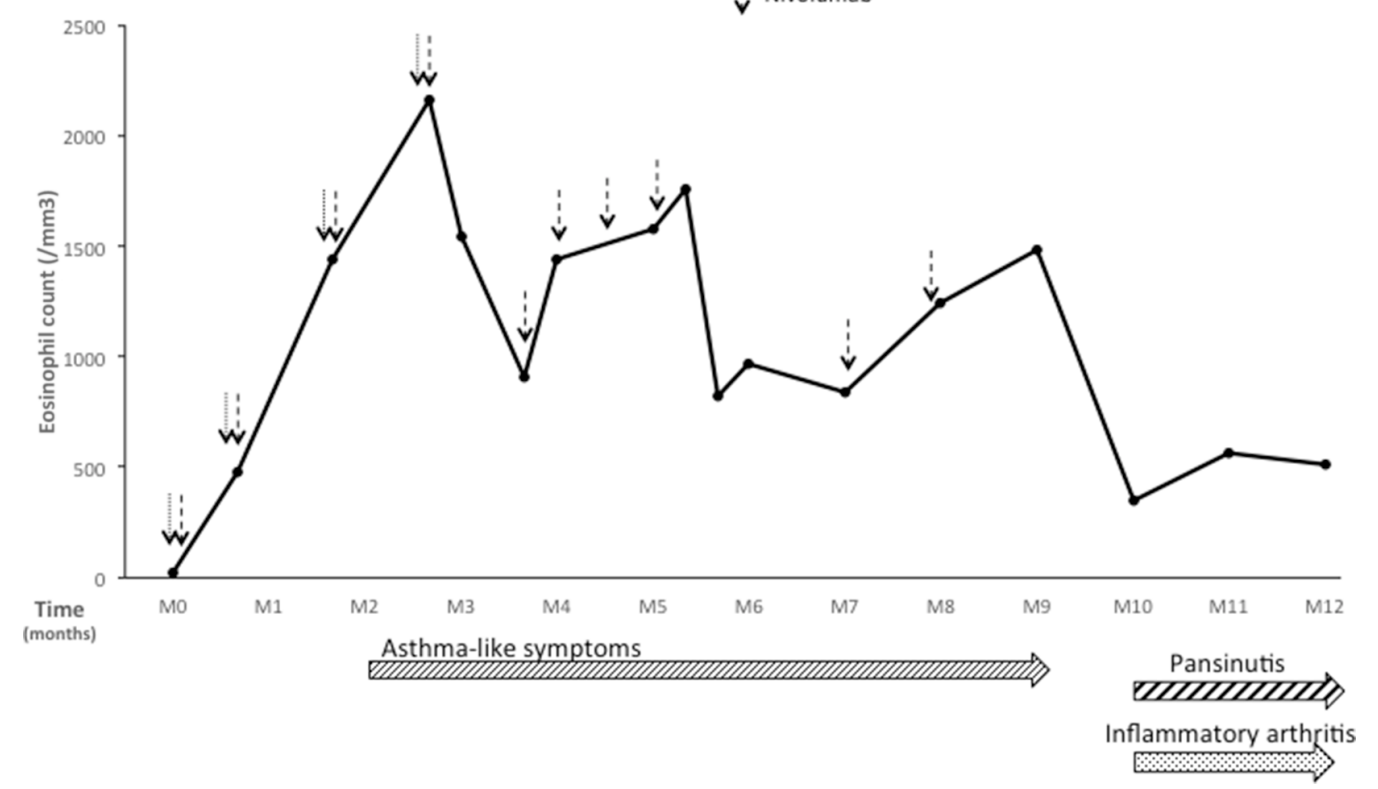

Figure 1 Timeline showing the relationship between serum eosinophil count and treatment with immune checkpoint inhibitors (double arrow: nivolumab $1 \mathrm{mg} / \mathrm{kg}$ and ipilimumab $3 \mathrm{mg} / \mathrm{kg}$ infusions; single arrow: nivolumab $3 \mathrm{mg} / \mathrm{kg}$ infusion). 
Kostine et $a l^{1}$ suggest that patients with rheumatic irAEs are more likely to respond to ICI, and increased eosinophil counts also correlate with improved survival in patients with metastatic melanoma treated with ICI, ${ }^{9}$ possibly due in part to the antitumour effect of eosinophil granulocytes. ${ }^{10}$ In this line, a complete tumour response persisted 12 months after nivolumab discontinuation in our patient.

Anissa Roger, ${ }^{1}$ Matthieu Groh, ${ }^{2}$ Gwenael Lorillon, ${ }^{3}$ Claire Le Pendu, ${ }^{2}$ Jeremy Maillet, ${ }^{4}$ Dimitri Arangalage ${ }^{5,6,7}$ Abdellatif Tazi, ${ }^{3,6,8}$ Celeste Lebbe, ${ }^{1,6,9}$ Barouyr Baroudjian, ${ }^{1}$ Julie Delyon, ${ }^{1,6,9}$ the PATIO group

'Department of Dermatology, AP-HP Hôpital Saint Louis, Paris, France

2Department of Internal Medicine, AP-HP Hôpital Saint Louis, Paris, France

${ }^{3}$ Department of Pneumology, AP-HP Hôpital Saint Louis, Paris, France

${ }^{4}$ Department of Rheumatology, AP-HP Hôpital Lariboisière, Paris, France

${ }^{5}$ Department of Cardiology, AP-HP Hôpital Bichat, Paris, France

${ }^{6}$ Université Paris Diderot, Paris, France

IINSERM U1148, Hôpital Bichat, Paris, France

${ }^{8}$ U1153 CRESS, Equipe de Recherche en Biostatistiques et Epidémiologie Clinique, Paris, France

9INSERM U976, Hôpital Saint Louis, Paris, France

Correspondence to Dr Julie Delyon, Department of Dermatology, AP-HP Hôpital Saint Louis, Paris Île-de-France, 75010 Paris, France; julie.delyon@aphp.fr

Contributors AR, MG and JD wrote the manuscript. BB, JD, GL, CLP, DA, JM and AT provided substantial information. All authors read and approved the manuscript.

Competing interests $\mathrm{CL}$ received research grants or honoraria from Roche, BMS, MSD, GSK, Novartis and Amgen.

Patient consent Obtained.

Provenance and peer review Not commissioned; internally peer reviewed.

Author note MG recently moved to the Department of Internal Medicine, National Referral Centre for Hypereosinophilic Syndromes (CEREO), Hopital Foch, Suresnes, France.

(c) Article author(s) (or their employer(s) unless otherwise stated in the text of the article) 2019. All rights reserved. No commercial use is permitted unless otherwise expressly granted.
To cite Roger A, Groh M, Lorillon G, et al. Ann Rheum Dis 2019;78:e82.

Received 31 May 2018

Accepted 6 June 2018

Published Online First 23 June 2018

\section{(}

http://dx.doi.org/10.1136/annrheumdis-2018-213895

Ann Rheum Dis 2019:78:e82. doi:10.1136/annrheumdis-2018-213857

\section{REFERENCES}

1 Kostine M, Rouxel L, Barnetche T, et al. Rheumatic disorders associated with immune checkpoint inhibitors in patients with cancer-clinical aspects and relationship with tumour response: a single-centre prospective cohort study. Ann Rheum Dis 2018;77:393-8.

2 Arnaud L, Lebrun-Vignes B, Salem JE. Checkpoint inhibitor-associated immune arthritis. Ann Rheum Dis 2019;78:e68.

3 Belkhir R, Burel SL, Dunogeant L, et al. Rheumatoid arthritis and polymyalgia rheumatica occurring after immune checkpoint inhibitor treatment. Ann Rheum Dis 2017;76:1747-50

4 Wolchok JD, Chiarion-Sileni V, Gonzalez R, et al. Overall survival with combined nivolumab and ipilimumab in advanced melanoma. $N$ Engl J Med 2017:377:1345-56.

5 Tocut M, Brenner R, Zandman-Goddard G. Autoimmune phenomena and disease in cancer patients treated with immune checkpoint inhibitors. Autoimmun Rev 2018;17:610-6.

6 Masi AT, Hunder GG, Lie JT, et al. The American College of Rheumatology 1990 criteria for the classification of Churg-Strauss syndrome (allergic granulomatosis and angiitis). Arthritis \& Rheumatism 1990:33:1094-100.

7 Bernard-Tessier A, Jeanville P, Champiat S, et al. Immune-related eosinophilia induced by anti-programmed death 1 or death-ligand 1 antibodies. Eur J Cancer 2017:81:135-7.

8 Cottin V, Bel E, Bottero P, et al. Revisiting the systemic vasculitis in eosinophilic granulomatosis with polyangiitis (Churg-Strauss). Autoimmun Rev 2017;16:1-9.

9 Delyon J, Mateus C, Lefeuvre D, et al. Experience in daily practice with ipilimumab for the treatment of patients with metastatic melanoma: an early increase in lymphocyte and eosinophil counts is associated with improved survival. Ann Oncol 2013:24:1697-703.

10 Carretero R, Sektioglu IM, Garbi N, et al. Eosinophils orchestrate cancer rejection by normalizing tumor vessels and enhancing infiltration of CD8(+) T cells. Nat Immuno 2015;16:609-17 